\title{
Application of Fuzzy Control Algorithms for Electric Vehicle Antilock Braking/Traction Control Systems
}

\author{
P. Khatun, C. M. Bingham, Member, IEEE, N. Schofield, and P. H. Mellor
}

\begin{abstract}
The application of fuzzy-based control strategies has recently gained enormous recognition as an approach for the rapid development of effective controllers for nonlinear time-variant systems. This paper describes the preliminary research and implementation of a fuzzy logic based controller to control the wheel slip for electric vehicle antilock braking systems (ABSs). As the dynamics of the braking systems are highly nonlinear and time variant, fuzzy control offers potential as an important tool for development of robust traction control. Simulation studies are employed to derive an initial rule base that is then tested on an experimental test facility representing the dynamics of a braking system. The test facility is composed of an induction machine load operating in the generating region. It is shown that the torque-slip characteristics of an induction motor provides a convenient platform for simulating a variety of tire/road $\mu-\sigma$ driving conditions, negating the initial requirement for skid-pan trials when developing algorithms. The fuzzy membership functions were subsequently refined by analysis of the data acquired from the test facility while simulating operation at a high coefficient of friction. The robustness of the fuzzy-logic slip regulator is further tested by applying the resulting controller over a wide range of operating conditions. The results indicate that ABS/traction control may substantially improve longitudinal performance and offer significant potential for optimal control of driven wheels, especially under icy conditions where classical ABS/traction control schemes are constrained to operate very conservatively.
\end{abstract}

Index Terms-Antilock braking system (ABS), braking, electric vehicle, fuzzy logic control, traction control.

\section{INTRODUCTION}

$\mathbf{F}$ UZZY logic, or the use of fuzzy sets, allows uncertain or inexact concepts to be represented. They derive from a grouping of elements into classes that do not possess sharply defined boundaries [1], [2]. Fuzzy set theory [3], on which fuzzy controllers are based, allows imprecise and qualitative information to be expressed in a quantitative manner. Over the years, fuzzy logic techniques have been applied to a wide range of systems, with many electronic control systems in the automotive industry, such as automatic transmission, engine control and antilock braking systems (ABSs) currently being pursued, particularly in the United States. These electronically controlled automotive systems realize superior characteristics through the use of fuzzy-logic-based control, rather than traditional control algorithms [4]. Moreover, since fuzzy logic

Manuscript received July 2, 2002; revised May 2, 2003.

P. Khatun, deceased, was with TH!NK Technologies, Ford Motor Company.

C. M. Bingham and N. Schofield are with the Department. of Electronic and Electrical Engineering, University of Sheffield, S1 3JD Sheffield, U.K. (e-mail: c.bingham@sheffield.ac.uk).

P. H. Mellor is with the Industrial Electronics Group, University of Bristol, Bristol, U.K.

Digital Object Identifier 10.1109/TVT.2003.815922 controllers deal with inexactness in a rigorous manner, they are effective at handling the uncertainties and nonlinearities associated with complex control systems such as traction control and antilock braking.

ABSs aim to control the wheel slip at an optimum value that can provide maximum tractive force during heavy braking. However, there are many complicating factors, especially for current IC-engined vehicles, where the brake torque at the wheel is nonlinearly related to the temperature of the brake linings, and the viscosity of the brake fluid changes with temperature and influences the rate at which brake pressure can be increased or decreased. An ABS or traction system must also be capable of coping with factors such as changes in road surfaces, vehicle loading, and steering. Moreover, wear patterns of braking components may disturb wheel speed sensors, and the variation in the adhesion of brake linings, brake hysteresis, tire inflation pressure, tire wear, and other external factors must be accommodated.

The design of model-based ABS or traction control (TC) schemes (along with those designed empirically) therefore tend to be nonlinear and time varying, and are complicated by the inclusion of high-order terms in the transfer functions. Also, to-date, a feature of many TC/ABSs for commercial IC-engine vehicles is that the realizable performance is often limited by the mechanical bandwidth of the active actuation systems (typically $20 \mathrm{~Hz}$ for ABS).

However, with the advent of electrically powered vehicles into the marketplace, the means for controlling drive-torque at much greater bandwidths is possible, with the torque produced by the electric motor being directly proportional to phase current [5]. Hence, some of the difficulties attributed to mechanical systems can be inherently addressed.

A further key obstacle to the development of robust ABS/TC has traditionally been the real-time estimation of the wheel-slip versus adhesion-coefficient characteristics for different tire types and road surface conditions [6]. Recent studies have investigated the application of observer/estimation schemes to obtain real-time data indirectly, with the extended Kalman filter (EKF) receiving particular attention [7]. However, published material to date is primarily derived from simulation studies, with practical implementation and validation of the techniques rare. Current commercial/passenger vehicles incorporating ABS/TC systems often employ lookup tables that are based on the results of experimental trials, and have been shown to provide adequate performance under many driving situations. This technique, however, is limited by the fixed structure of the control strategy, and is often "detuned" to accommodate worst 


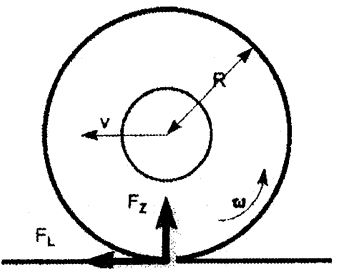

$\mathbf{v}$ : velocity of wheel

$\mathbf{R}$ : radius of wheel

$\mathbf{F}_{\mathbf{z}}$ : wheel load

$\mathbf{F}_{\mathbf{L}}$ : longitudinal force

$\omega$ : angular velocity of wheel

Fig. 1. Wheel model [8].

case scenarios, for example, traction control in icy conditions with old tires. Consequently, suboptimal wheel-slip characteristics are imposed for most driving conditions. Although this constitutes the safest criteria to adopt for design purposes, it can impose conservative limits on the traction available under higher $\mu$ surface conditions. Hence, maximum benefits of the traction controller are often not realized due to the classical control strategies used for design. The application of fuzzy-logic-based control algorithms is therefore investigated and discussed in this paper, along with a review of how to employ such methodologies to develop more robust and efficient ABS/traction control systems.

\section{TRACTION CONTROL/ANTILOCK BRAKING SySteMS}

The tractive force between a tire and the road surface (Fig. 1) is proportional to the normal load $F_{z}$, the constant of proportionality being termed the adhesion coefficient $\mu$. The adhesion coefficient $\mu$ is the ratio of tire brake force at the tire road interface and the normal load acting on the tire

$$
\mu=\frac{F_{L}}{F_{Z}} .
$$

The value of $\mu$ is highly dependent on tire characteristics (compound, wear, aging, etc.) and road surface conditions (dry, icy, gravel, tarmac, etc.), although it can be primarily regarded as a function of the relative slip $\sigma$ between the two contacting surfaces. By definition, slip is the normalized difference between the wheel speed and vehicle speed at the contact point

$$
\sigma=\frac{v-R \omega}{v}
$$

where $v$ is the vehicle velocity, $R$ is the effective radius of the driven wheel, and $\omega$ is the angular velocity of the wheel. Typical $\mu-\sigma$ characteristics are shown in Fig. 2.

It is clear from Fig. 2 that increasing slip can increase the tractive force between the tire and road surface by virtue of an increase in $\mu$. However, once the peak of the characteristic $\mu_{\max }$ is encountered, any further increase in slip will reduce traction and consequently induce an unstable acceleration of the wheel until the drive torque is reduced.

The objective of an ABS is to manipulate the torque applied to the driven wheels in order to limit the slip $\sigma$ between the road surface and the tire, and consequently, only operate within the stable region of the $\mu-\sigma$ characteristic. A major obstacle in the practical design of ABS control schemes is the determination, in real time, of $\mu-\sigma$ characteristics, i.e., $\mu_{\max }$ and $\sigma$. For example, Table I shows typical values of slip required to obtain the maximum adhesion coefficient for various road conditions.

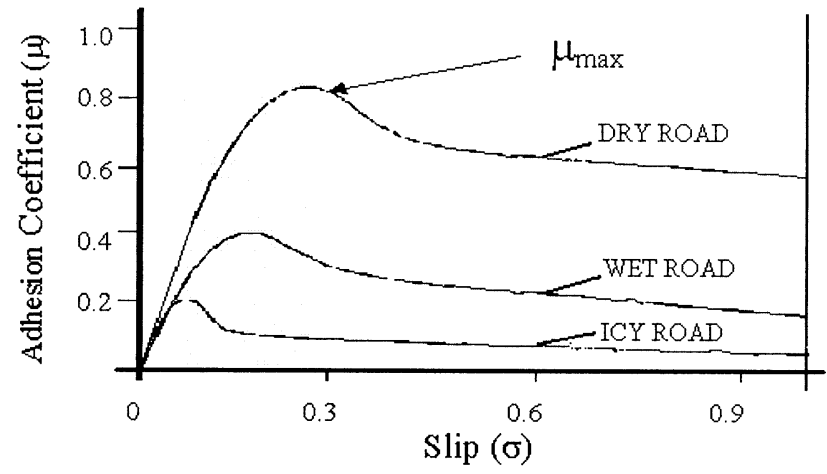

Fig. 2. Example $\mu$-slip characteristics for various road conditions [9].

TABLE I

MAX VALUES OF $\mu$ FOR VARIOUS ROAD SURFACES [9]

\begin{tabular}{c|c|c}
\hline $\begin{array}{c}\text { Road } \\
\text { condition }\end{array}$ & $\begin{array}{c}\text { Max. adhesion } \\
\text { coefficient }\left(\mu_{\max }\right)\end{array}$ & $\begin{array}{c}\text { Optimum } \\
\text { slip }(\boldsymbol{\sigma})\end{array}$ \\
\hline Dry Road & 0.85 & 0.35 \\
\hline Wet Road & 0.4 & 0.2 \\
\hline Icy Road & 0.2 & 0.1 \\
\hline
\end{tabular}

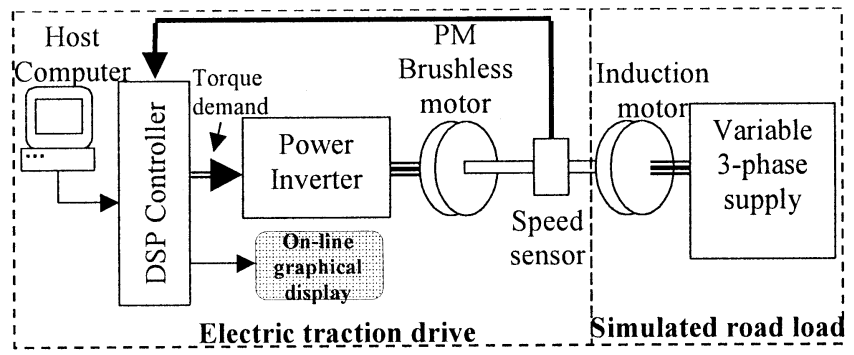

Fig. 3. DSP-based experimental test facilities.

To overcome this problem, many manufacturers employ a slip-limiting control scheme to account for "worst case" conditions, typically icy roads. Although this constitutes the safest criteria to adopt for design purposes, it can impose conservative limits on the traction available under less severe conditions [10]. A second obstacle often encountered by designers of TC/ABS schemes is the availability of appropriate test-tracks/skid-pans or other experimental rolling road facilities to evaluate proposed algorithms and provide repeatable conditions for comparative studies.

\section{EXPERIMENTAL TEST FACILITY}

To develop the fuzzy control algorithms, a low-cost experimental test bench is employed. The test facility, illustrated in Fig. 3, is composed of an electric traction drive connected to a standard three-phase induction machine, which is used to mimic the road load.

The experimental electric traction drive is a 6-kW continuous, $12-\mathrm{kW}$ peak rated brushless permanent magnet motor supplied via a power controller from a $72-\mathrm{V}$ high-performance nickel cadmium traction battery. The power electronic controller is constructed using three independent MOSFET/IGBT H-bridges 


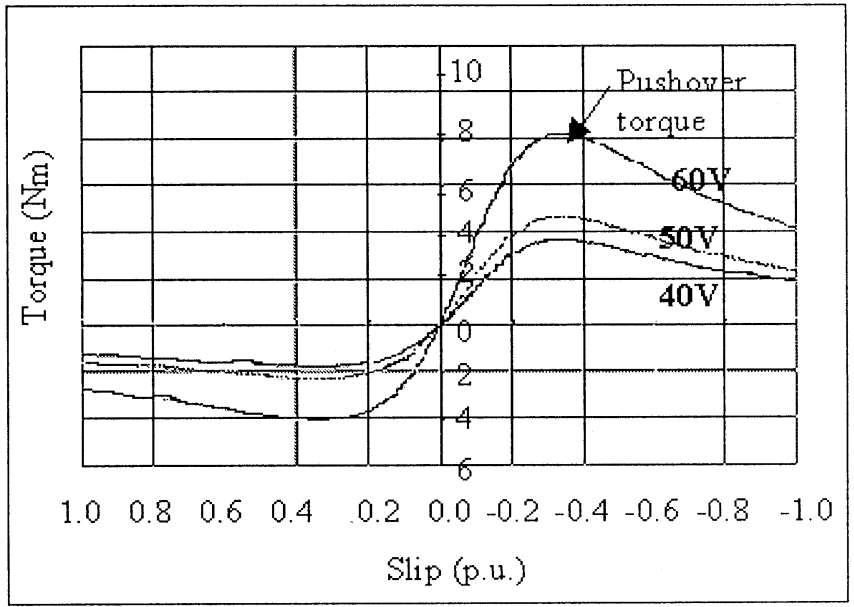

Fig. 4. Induction machine torque-slip characteristics.

[11] and is capable of full four-quadrant operation. The electrical machine and power converter has a combined efficiency of $92 \%$ at rated power. In the prototype electric vehicle, the traction motor is connected to the wheels via a step-down gearstage. However, for the proposed test-bench, the brushless permanent magnet motor is directly coupled to a $2.2-\mathrm{kW} 50-\mathrm{Hz}$ three-phase six-pole induction machine, with the angular velocity of the connecting shaft being monitored via an optical shaft encoder interface. The induction motor is connected to the utility supply via a variac, allowing the supply voltage to be adjusted. To facilitate the implementation of the developed control schemes, a custom state-of-the-art digital signal-processing (DSP) hardware platform has been designed, based on the TMS320C31 floatingpoint processor, to integrate the system control algorithms with sensor interfaces, man-machine interfaces, monitoring, and supervisory tasks. A foreground/background distribution of "nontime-critical" and "time-critical" functions is employed to ensure the controller remains a high-integrity process.

The key operating principle of the proposed test facility for TC/ABS investigations is based on the correspondence between $\mu-\sigma$ characteristics of tire/road interactions (Fig. 2) and the torque-slip characteristics of the induction machine when operated as a generator (Fig. 4). In line with convention, induction machine slip is shown in terms of per-unit (p.u.) values, with reference to the synchronous frequency $\omega_{\text {sync }}$, and provides a natural analogy with the definition of tire-road slip; see Fig. 5.

The brushless permanent magnet (PM) motor acts as the prime mover and drives the induction machine into the generator region of operation, thereby inducing a negative slip with respect to synchronous speed (1000 rpm in this case). As the torque demand to the PM machine is raised further, the slip becomes increasingly negative, and the induction machine develops an opposing torque, eventually resulting in a stable steady-state condition.

This procedure can continue up to the pushover torque limit of the induction machine. A subsequent increase in applied torque will then further decrease the slip and force the system into an unstable operating regime where the induction machine is not able to oppose the applied torque, and a runaway condition is encountered with the brushless motor accelerating uncontrol-

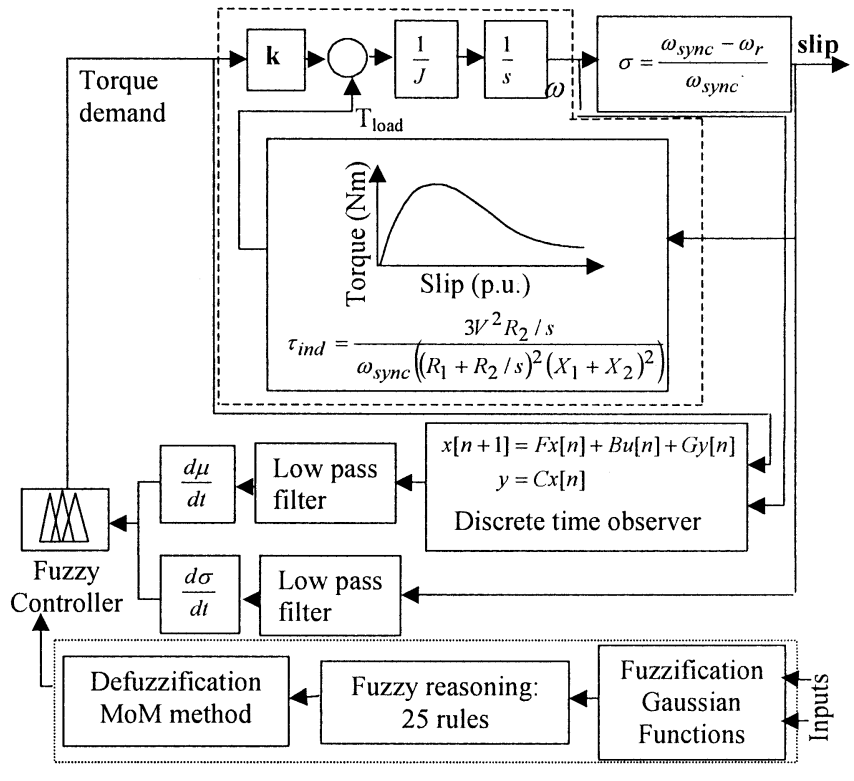

Fig. 5. Fuzzy-logic-based traction control scheme.

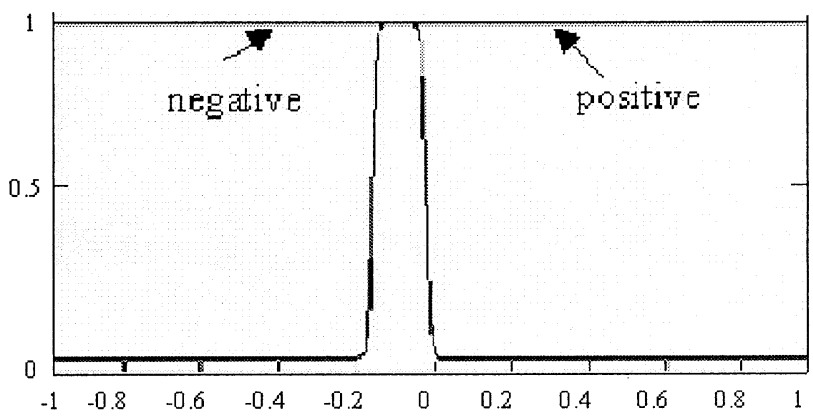

(a)

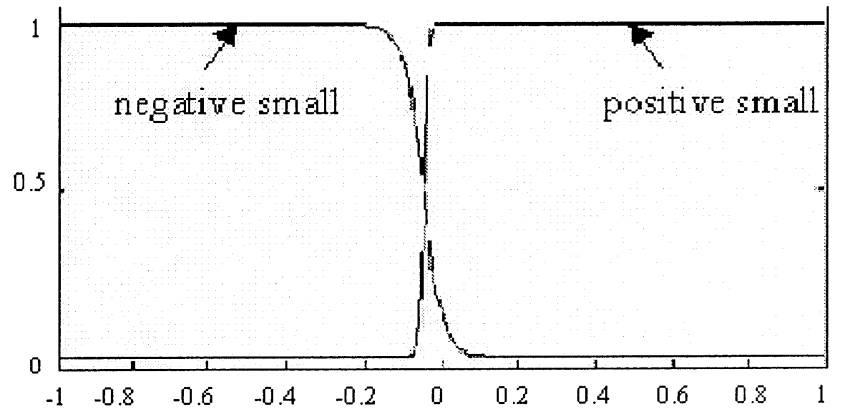

(b)

Fig. 6. Membership functions for (a) observed load torque and (b) slip.

lably. This is analogous to the classical $\mu-\sigma$ characteristics encountered in traction control systems when the slip at $\mu_{\max }$ is exceeded. An algorithm that automatically controls the torque developed by the brushless PM motor to maintain the induction machine in the "stable-slip" region of operation is therefore also likely to be a good candidate to control the torque applied to the wheels of an electric vehicle to maintain tractive force in the stable region of the $\mu-\sigma$ characteristic. The torque-slip characteristic can be obtained from the standard equivalent circuit of an induction machine

$$
\tau_{\text {ind }}=\frac{3 V^{2} R_{2} / s}{\omega_{\text {sync }}\left(\left(R_{1}+R_{2} / s\right)^{2}+\left(X_{1}+X_{2}\right)^{2}\right)}
$$




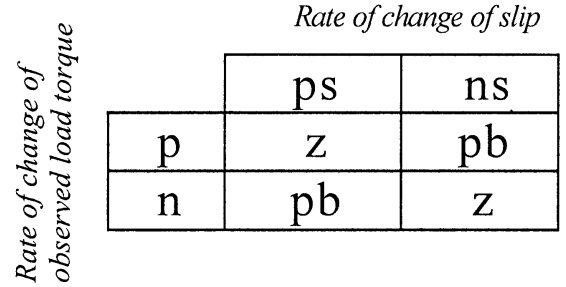

Fig. 7. Rule map for fuzzy traction controller.

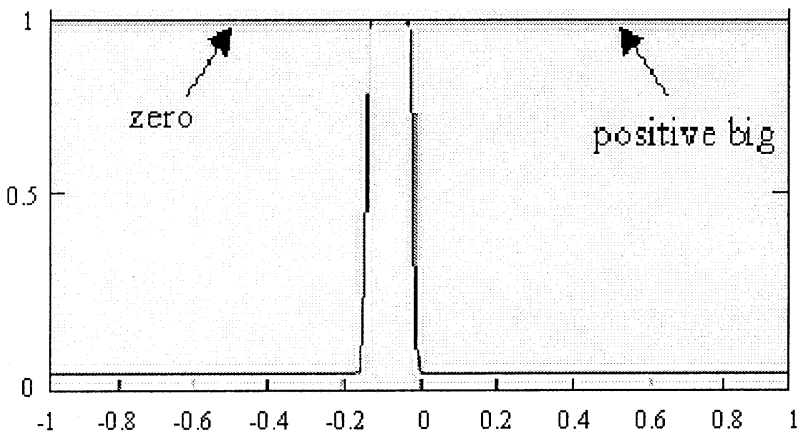

Fig. 8. Membership function for induction machine torque demand.

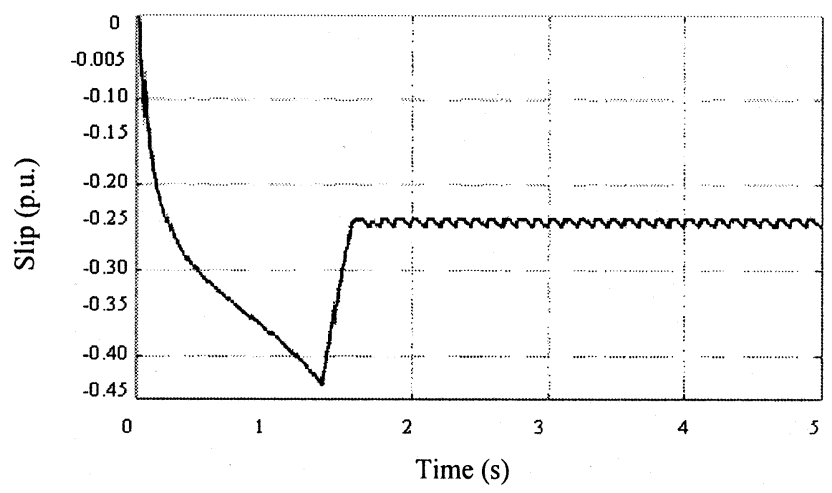

Fig. 9. Simulation results for road conditions with fuzzy logic control.

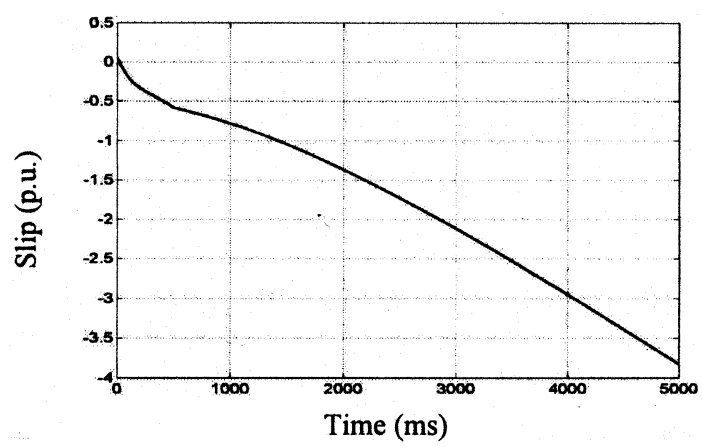

Fig. 10. Simulation results for road conditions without fine "tuning" of fuzzy controller.

where $V$ is the applied voltage, $R_{1}$ is the Thevenin equivalent stator resistance, $R_{2}$ is the referred rotor resistance, $s$ is the slip, $\omega_{\text {sync }}$ is the synchronous speed, $X_{1}$ is the Thevenin equivalent stator reactance, and $X_{2}$ is the referred rotor reactance.

The magnitude and form of the torque-slip characteristics are dependent upon the supply voltage and impedance; by adjusting these, a variety of tire/road conditions can be readily simulated. It is noted that, due to the unsymmetrical nature of induction

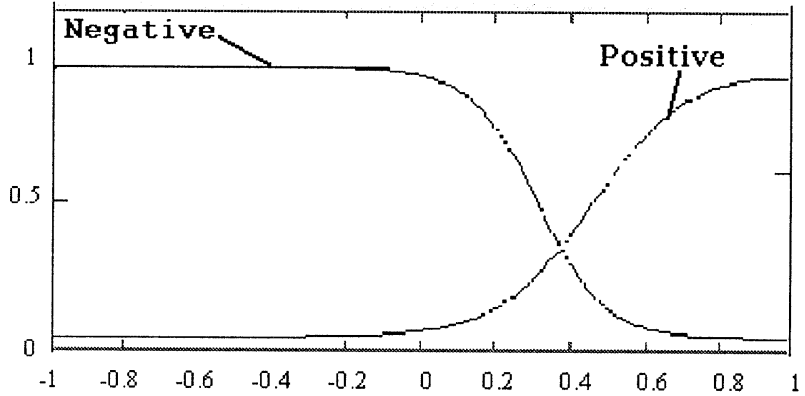

(a)

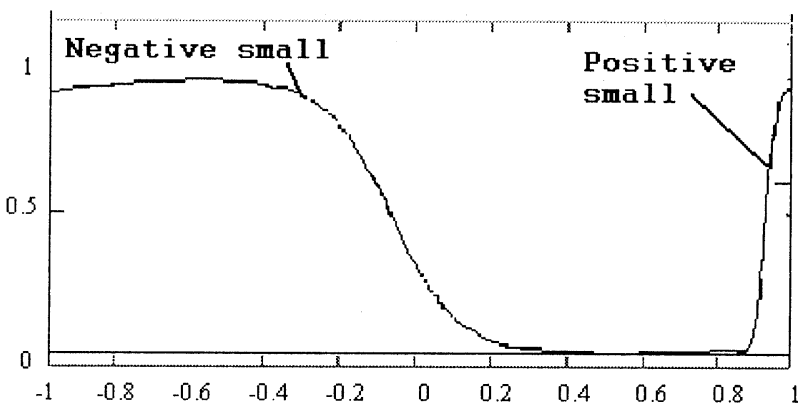

(b)

Fig. 11. "Tuned" membership functions for (a) observed load torque and (b) slip.

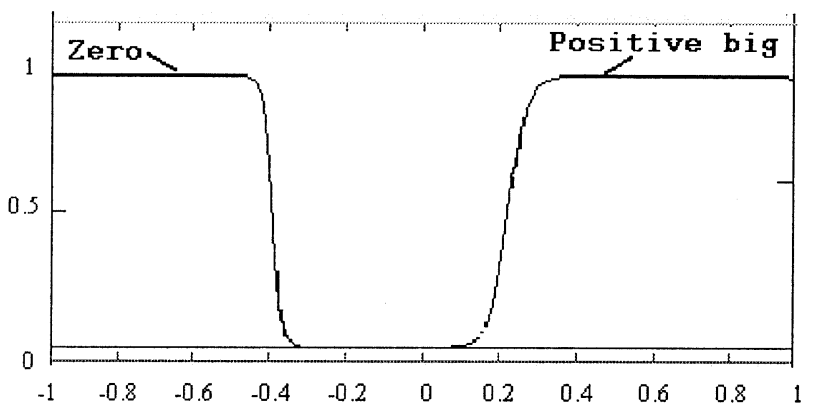

Fig. 12. "Tuned" membership function for output torque demand.

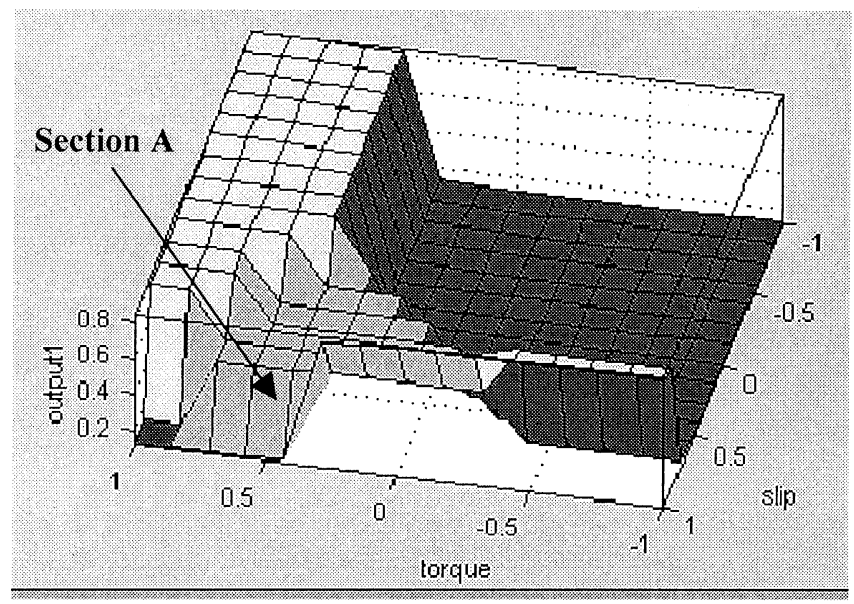

Fig. 13. Three-dimensional surface mesh of fuzzy membership function distribution.

machine characteristics, different parameters for exciting the induction machine are required to simulate ABSs and TC systems. 

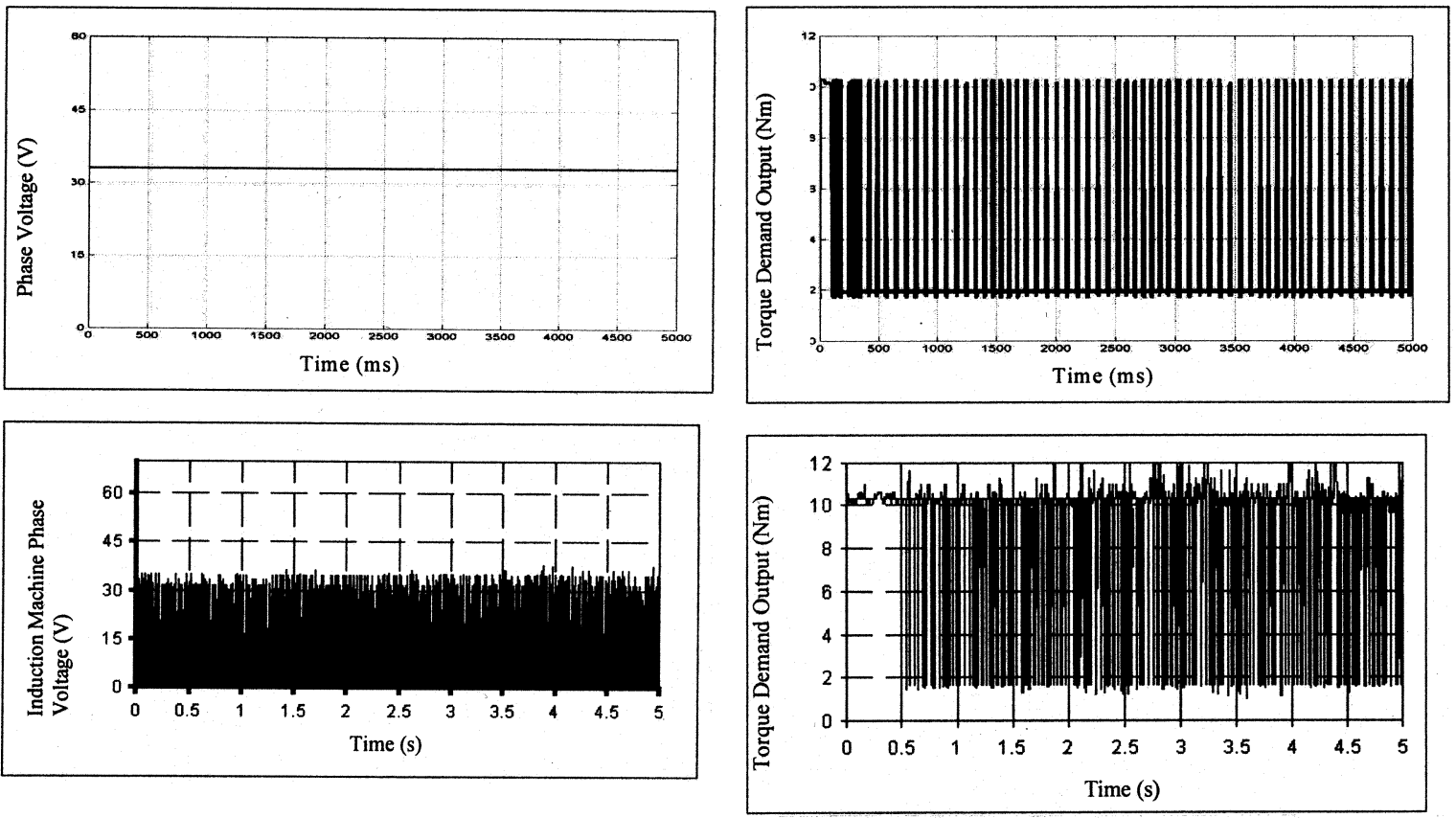

(a)

(b)
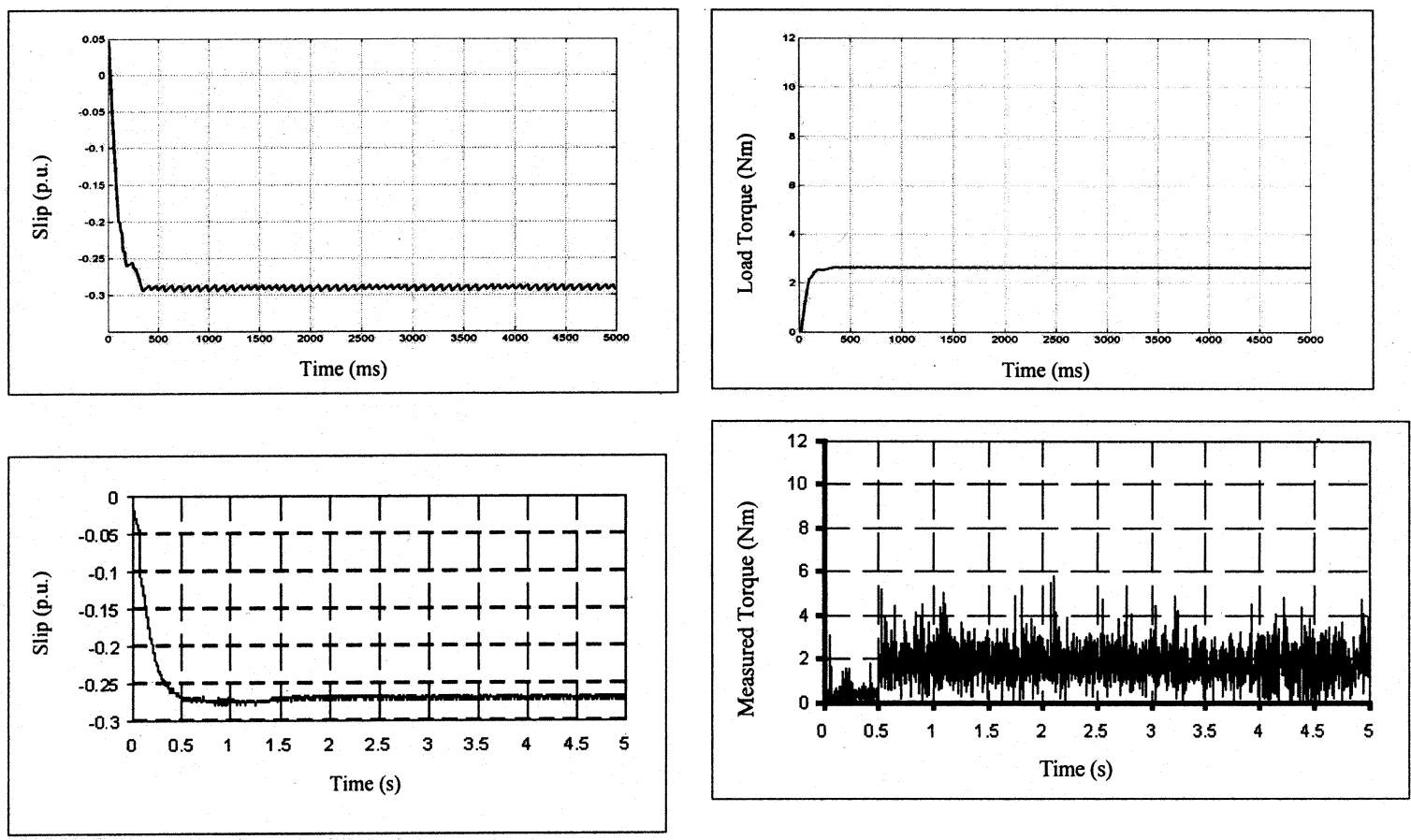

(c)

(d)

Fig. 14. Simulation and measured results at $33 \mathrm{~V}$ phase voltage, which is analogous to an icy surface of $\mu=0.2$.

\section{FuZZY LOGIC ABS/TraCtion CONTROL}

To address the aforementioned issues, a fuzzy-based methodology is employed to develop control algorithms. One of the strengths of fuzzy systems is their ability to express a confidence in reasoning results. Recent studies have shown that fuzzy systems are part of a universal class of approximators of continuous functions.

The key advantage of fuzzy control is that there are many instances where TRUE and FALSE or ON and OFF fail to describe a given situation. These cases require a sliding scale where variables can be measured as PARTLY ON or MOSTLY TRUE and PARTLY FALSE [4].

Traditional set theory is based on bivalent logic where an object either is a member of a set or is not. However, with fuzzy logic, an object can be a member of multiple sets with a different degree of membership in each set. A degree of membership in a set is based on a scale from 0 to 1 , with " 1 " being complete membership and " 0 " being no membership. Hence, by applying fuzzy logic control to this discrete-time traction con- 

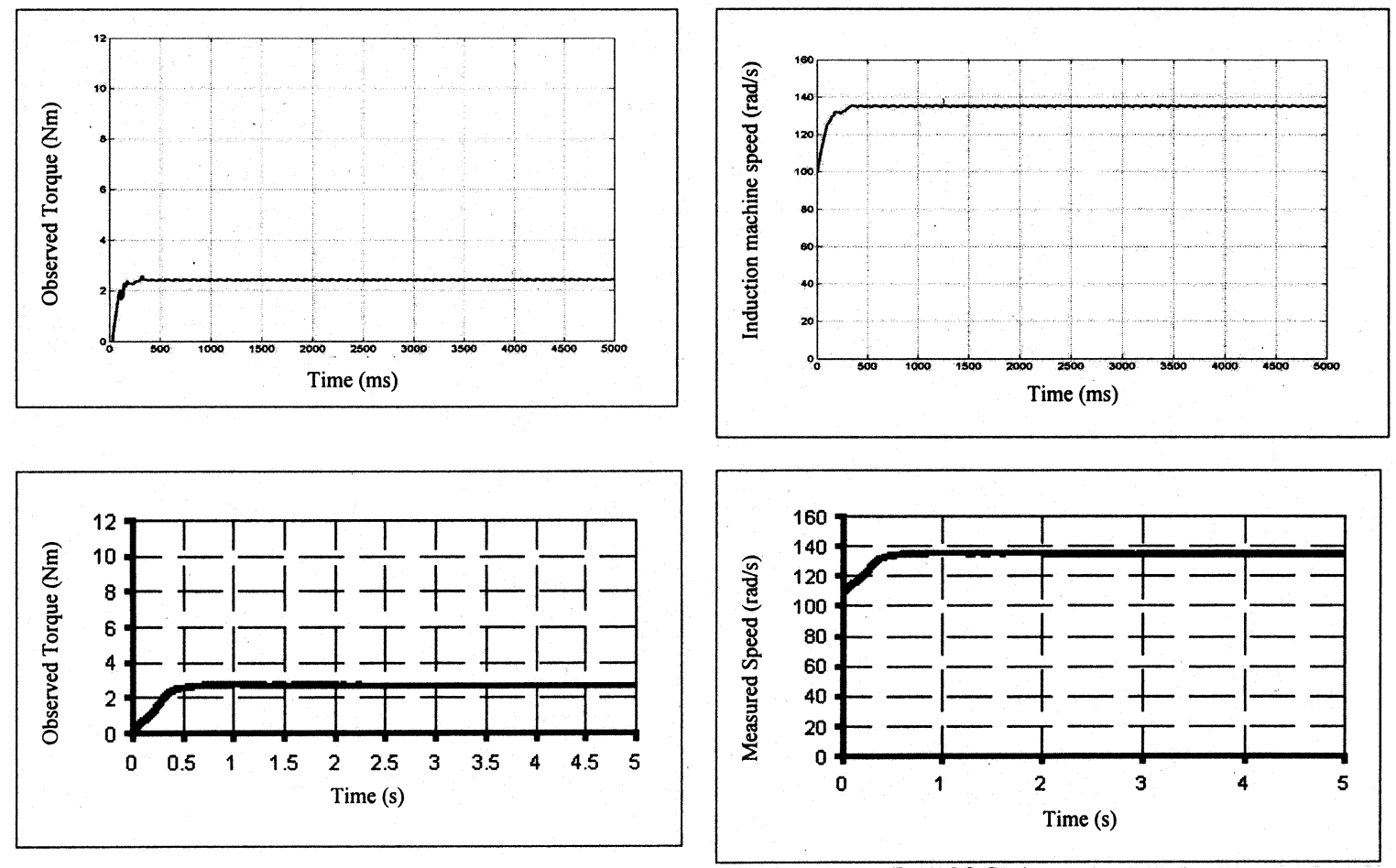

(e)

(f)

Fig. 14. (Continued.) Simulation and measured results at $33 \mathrm{~V}$ phase voltage, which is analogous to an icy surface of $\mu=0.2$.

trol system, an output (the torque demand) is calculated based on the amount of membership the input signals (observed load torque and slip, in this case) have in the configured fuzzy sets; see Fig. 5. Again, with reference to Fig. 5, the depicted observer is essentially a discrete-time filter that compares measured information at its inputs with model-based calculated data of the observed process, to provide convergent estimates of otherwise unmeasurable system states. In this case, observing the load torque $T_{\text {load }}$ presented to the induction machine is analogous to estimating the tractive force between the tire and the road surface on a vehicle, and hence, is directly related to $\mu$. A detailed account of the design of the observer for this type of system, in the digital domain, is given in [14]. Given that slip is readily measurable, the role of the observer is to facilitate the determination of when the pushover torque (or, equivalently, $\mu_{\max }$ on a vehicle) is exceeded, based only on measurements of slip and demanded torque. This condition depicts the transition between the stable and unstable regions of the $\mu-\sigma$ characteristic and is obtained by noting the occurrence of a sign change of $\mathrm{d} \mu / \mathrm{d} \sigma$. Upon a transition into the unstable region, the torque demand is reduced until operation in the stable region is again detected, at which time the demanded torque is once again allowed to be developed. In this manner, the characteristically high bandwidth afforded by the electric traction system allows the ABS/traction controller to operate about the peak of the $\mu-\sigma$ characteristic, irrespective of driving conditions. Detecting the transition between the stable and unstable regions, however, is hindered by measurement noise and modeling error when designing the observer and presents a key driver for employing fuzzy-based control techniques for this application.
The system inputs are therefore exposed to three key transformations before becoming a system output, as shown in Fig. 5, which includes the transformation process of fuzzification, fuzzy rule association, and defuzzification.

To establish the two appropriate input fuzzy membership functions and the rule base, a thorough investigation of the ABS braking process has been undertaken. In this case, the rate of change of observed load torque and the rate of change of slip are chosen and are fuzzified into membership functions, as shown in Fig. 6(a) and (b).

Initially, Gaussian functions have been employed to investigate the ABS braking process; however, experimental trials have ultimately shown that functions based on polysigmoid curves can provide enhanced performance characteristics.

For the slip, the universe of discourse was chosen between 0 and $100 \%$, since during the operation of an induction machine at zero slip the system is stable and at $100 \%$ the system is unstable. This is analogous to the braking process of a vehicle, where a wheel can rotate freely with slip at zero or be locked at slip equal to one.

Both inputs, slip and observed load torque, are fuzzified into two membership functions. A smaller number of membership functions causes less sensitive control, but better computation speed, and vice versa. Therefore, it is very important to understand and test the influence of the number of membership functions, as a compromise always exists between execution speed and sensitivity of the fuzzy controller.

For testing purposes, the fuzzy-logic-based traction control scheme simply has two inputs (slip and load torque) to the controller and one output (torque demand), with four possible rules. 

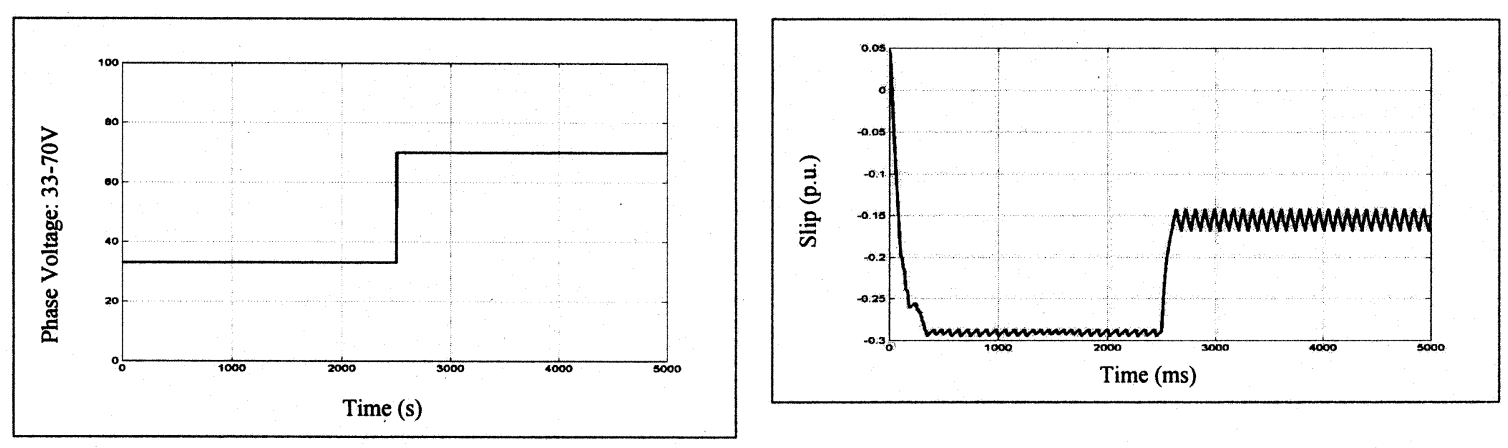

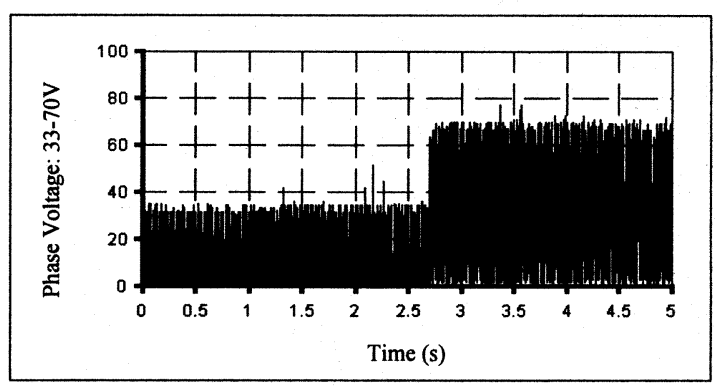

(a)
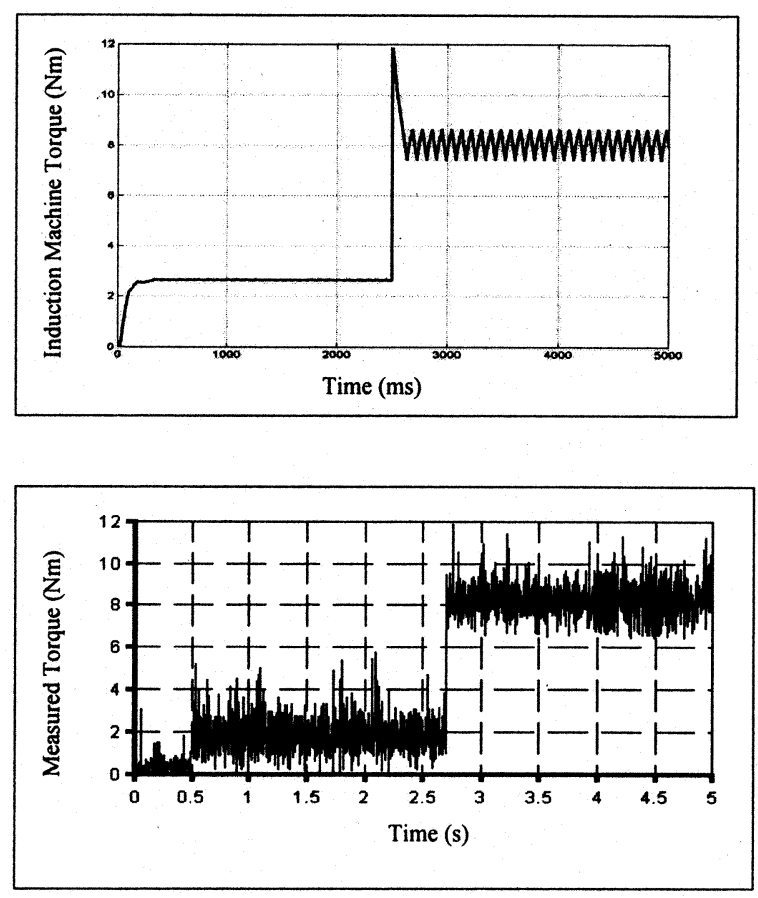

(c)

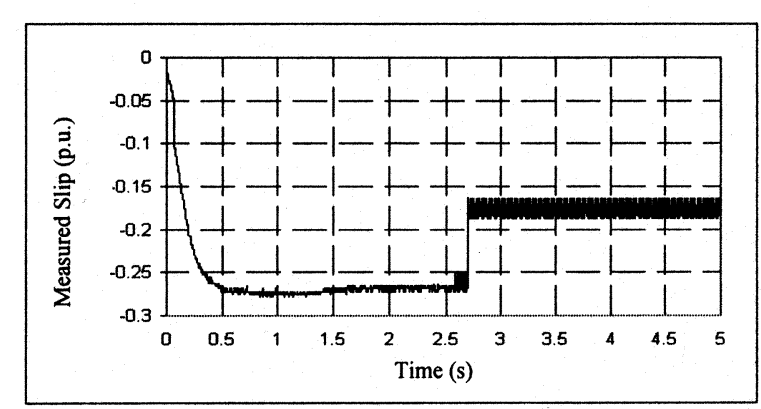

(b)
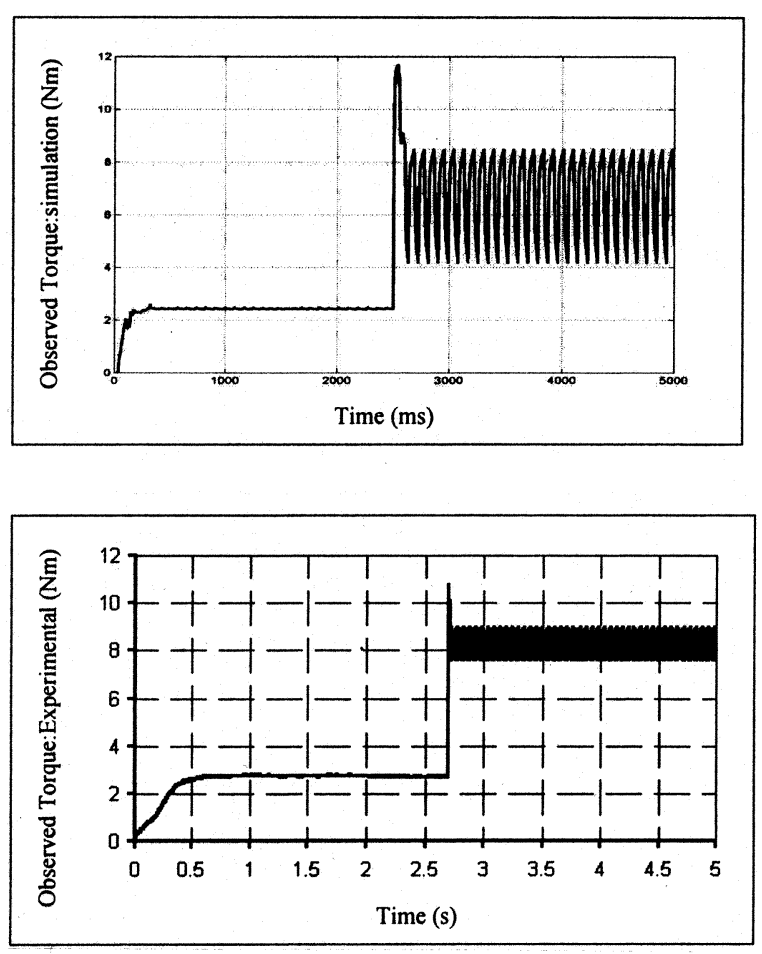

(d)

Fig. 15. Simulation and measured results between 33- and 70-V phase voltage, which models a vehicle travelling from an icy to a dry road surface.

All four rules are used for the controller and are determined from the fuzzy rule map shown in Fig. 7. The four rules cover each state of input combinations (p: positive, n: negative, ps: positive small, and ns: negative small) during the braking process. The output (z: zero or pb: positive big) of the fuzzy controller is the torque demand (or, in the case of a vehicle, the tractive force). The fuzzy membership functions for the output, torque demand, are shown in Fig. 8.
The membership functions, shown in Fig. 6, were created by experimentally testing the relationship between slip and torque of the induction machine. A fuzzy controller consists of fuzzification, rulebase, and defuzzification procedures. Fuzzification, that is, creating fuzzy inputs from crisp inputs, is accomplished using the membership functions in Fig. 6(a) and (b). The fuzzy output is generated using the rulebase shown in the rulemap in Fig. 7. A crisp output is generated from the fuzzy output by de- 

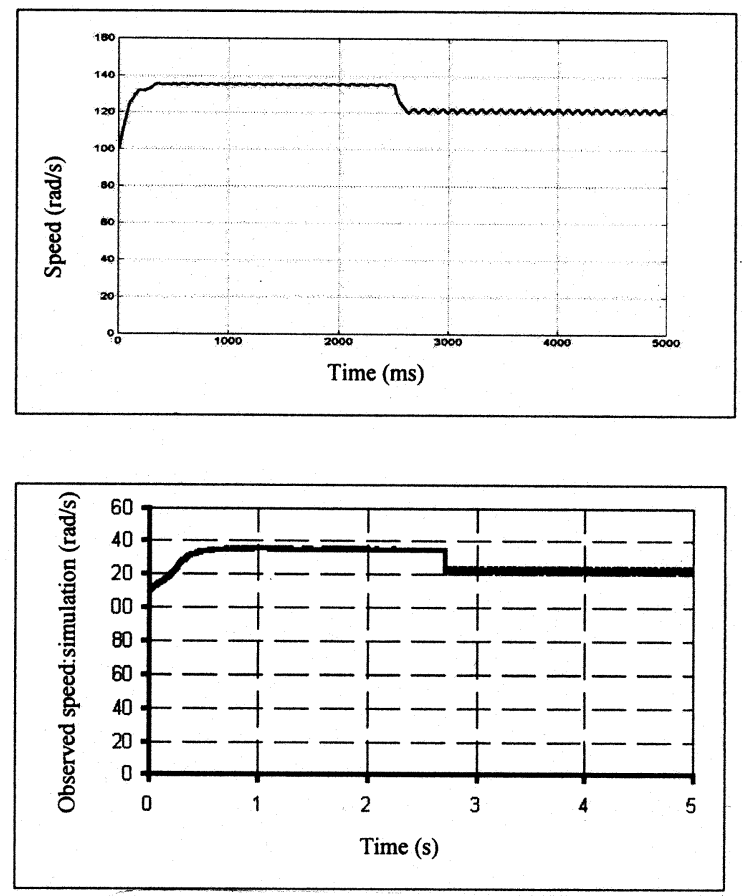

Fig. 15. (Continued.) Simulation and measured results between 33- and 70-V phase voltage, which models a vehicle travelling from an icy to a dry road surface.

fuzzification using the membership functions shown in Fig. 8. This is the basic fuzzy control algorithm for a traction control system.

\section{RESUlTS}

The fuzzy control scheme has been implemented with an experimental setup (at $60-\mathrm{V}$ phase voltage) analogous to a vehicle travelling on a dry road surface. Fig. 9 shows the simulated performance of the system. By comparison with classical "bang-bang" slip control schemes, where the slip is generally limited to -0.1 p.u. for dry surfaces [12], it can be seen that the fuzzy scheme automatically adapts the slip control algorithm, identifies the unstable region of the torque-slip curve and reduces the slip. Eventually the slip stabilizes at -0.25 p.u., which is similar to the $\mu-\sigma$ characteristic of a dry road surface (where the peak slip is between -0.25 and -0.35 p.u.).

However, without fine "tuning" of the fuzzy controller, the system can be shown to be unstable at lower phase voltages (35 $\mathrm{V})$, analogous to a very icy road surface conditions at -0.1 p.u. slip (Fig. 10).

Hence, some "tuning" of the input and output membership functions (Figs. 11 and 12), e.g., by perturbing the membership functions, is required; the controller hence being optimized for lower values of adhesion. This is shown graphically on the threedimensional surface mesh plot of Fig. 13, where an increase in the distribution of Section A provides a more robust controller.

As a result, the fuzzy controller is further tuned to ensure optimum control for a vehicle travelling on low $\mu$ surfaces (or 33-V induction machine phase voltage), which is demonstrated by the results in Fig. 14, that depict both simulated and measured data, respectively (in each case, the upper subfigure relates to sim- ulated results while the lower corresponds to measured data). From Fig. 14(b), it can be seen the demanded drive train shaft torque of $12 \mathrm{Nm}$ (maximum) was developed by the PM brushless machine by connection to the induction machine load. Furthermore, results shown in Fig. 14(c) demonstrate that the fuzzy controller's robustness is sufficient to keep the induction machine from operating in the unstable torque-slip region, especially at low values of adhesion. It is notable that the measured load torque [Fig. 14(d)] was derived from the measured phase current, using the relationship $T \propto k I$, where the constant $k$ is given by $0.23 \mathrm{Nm} / \mathrm{A}$. Finally, Fig. 14(e) illustrates the observed torque that is derived by the discrete time-based observer [13], whose characteristics show a similar dynamic response as that of the measured load torque.

To provide dynamic $\mu-\sigma$ characteristics to simulate a wide range of road conditions, real-time variation of phase voltage applied to the induction machine can be employed. Consequently, it is possible to observe the fuzzy load torque (or $\mu$ ) over a time period of $5 \mathrm{~s}$, while the phase voltage is varied between 33 and $70 \mathrm{~V}$ (Fig. 15). This transient is analogous to the vehicle travelling from an icy road surface to a dry road surface. It can be seen that operation transfers from an observed torque $(\approx 2.2 \mathrm{Nm})$ relating to an equivalent $\mu_{\max }$ for icy conditions to a torque $(\approx 8$ $\mathrm{Nm}$ ) relating to an equivalent $\mu_{\max }$ for a dry road surface. The oscillations shown in Fig. 15(d) are indicative of the repetitive transfer between the stable and unstable regions of the $\mu-\sigma$ characteristic, about $\mu_{\max }$, driven purely from information provided by the observer, and the high-frequency modulation of the phase voltage used as a means of controlling torque demand (analogous to controlling the torque developed at the driven wheels of a vehicle) [Fig. 15(a)] derived from fuzzy scheme.

Thus, from the measured data, it can be seen that the incorporation of the fuzzy-based discrete-time observation technique allows the dynamic $\mu-\sigma$ characteristics to be obtained without a-priori knowledge of $\mu_{\max }$.

\section{CONCLUSION}

A key aim of the research has been to establish a practical understanding of the ability of fuzzy logic schemes to control ABSs with significant, time-varying nonlinear dynamics. From the results, it can be clearly seen that the fuzzy controller is capable of compensating for the complex nonlinear behavior. The robustness of the fuzzy controller has ultimately been shown to be impressive, with the ability to adjust to varying road surfaces being demonstrated. Additionally, the results suggest that fuzzy-logic-based ABS/traction control could substantially improve longitudinal performance and offer significant potential for optimal control of driven wheels, particularly in icy road conditions.

\section{ACKNOWLEDGMENT}

The authors would like to acknowledge the U.K. Engineering and Physical Sciences Research Council for providing a research studentship for P. Khatun, to whose memory this paper is now respectfully dedicated. 


\section{REFERENCES}

[1] P. Pedrycz and F. Gomide, An Introduction to Fuzzy Sets. Cambridge, MA: MIT Press, 1998.

[2] A. Kandel, Fuzzy Mathematical Techniques with Applications. Reading, MA: Addison-Wesley, 1998.

[3] L. A. Zadeh, "Fuzzy Sets," Inform. Contr., 1965.

[4] P. M. De Koker, J. Gouwes, and L. Pretorius, "Fuzzy control algorithm for automotive traction control systems," Proc. Melcon '96, vol. 1, pp. 226-229, 1996

[5] S. Howell, "Traction control for an electric vehicle,", M.Eng. project, Dept. of EEE, Univ. of Sheffield, 2000.

[6] J. L. Harned, L. E. Johnstine, and G. Scharpf, "Measurement of tire brake force characteristic as related to a wheelslip (antilock) control system," SAE Trans., vol. 78, no. 69214, 1969.

[7] L. R. Ray, "Non linear system and tire force estimation for advanced vehicle control," IEEE Trans. Contr. Syst. Technol., vol. 3, no. 1, pp. $117-124,1995$.

[8] R. Klien. (1999) Antilock braking system and vehicle speed estimation using fuzzy logic. [Online]. Available: http://www.fuzzytech.com/

[9] V. A. Constantin, "Fuzzy logic in automotive engineering," presented at the Embedded Systems Conf., 1996

[10] B. J. Robinson, “A study of various car antilock braking system," Dept. of Transport and Road Research Laboratory, U.K., Res. Rep. 340, 1991.

[11] P. H. Mellor, N. Scofield, and P. J. Hor, "Brushless permanent magnet drives for all-electric racing karts," in Proc. 29th Int. Symp. Automotive Technology Automation, 1996.

[12] P. Khatun, C. M. Bingham, N. Schofield, and P. H. Mellor, "Antilockbraking/traction control for a high-performance all-electric racing vehicle," presented at the 41st Int. Conf. PCIM for Power Conversion and Intelligent Motion, Nuremburg, June 22-24, 1999.

[13] P. Khatun, C. M. Bingham, and P. H. Mellor, "Comparison of control methods for electric vehicle antilock braking/traction control systems," presented at the SAE 2001 World Congr., Paper 2001010 596, Detroit, MI, Mar. 2001

[14] P. Khatun, C. M. Bingham, P. H. Mellor, and N. Schofield, "Discrete-time ABS/TC test facility for electric vehicles," in Proc. EVS-16, China, 1999.

P. Khatun received the B.Eng. (Hons.) in integrated engineering with automotive studies from Sheffield Hallam University, U.K., in 1997.

She undertook research into fuzzy-based antilock braking and traction control systems in the Department. of Electronic and Electrical Engineering, University of Sheffield, U.K. She subsequently became a Product Design Engineer with TH!NK Technologies, a division of Ford Motor Company, in 2001, where she remained until being fatally injured in a tragic road accident in Autumn 2002.

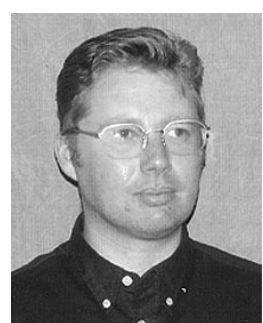

C. M. Bingham (M'00) received the B.Eng. degree in electronic systems and control engineering, from Sheffield City Polytechnic, Sheffield, U.K., in 1989, the M.Sc. (Eng.) degree in control systems engineering from the University of Sheffield in 1990, and the Ph.D. degree from Cranfield University, U.K., in 1994, for research on control systems to accommodate nonlinear dynamic effects in aerospace flight-surface actuators.

He remained there as a Postdoctoral Researcher until taking up a research position at the University of Sheffield. Since 1998, he has been a Lecturer in the Department of Electronic and Electrical Engineering, University of Sheffield. His current research interests include traction control/antilock braking systems for electric vehicles, electromechanical actuation of flight control surfaces, control of active magnetic bearings for high-speed machines, sensorless control of brushless machines, and the high-speed modeling, analysis, and design of resonant power converters.

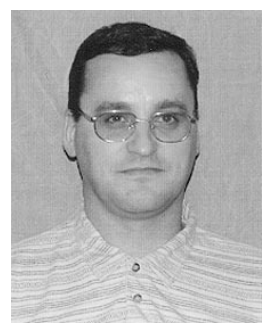

N. Schofield received the B.Eng. degree in electrical power engineering and the Ph.D.degree from the University of Sheffield, Sheffield, U.K., in 1990 and 1997, respectively.

His doctoral research was on the field-weakening of brushless permanent-magnet traction machines. During 1993-1995, he was a Senior Experimental Officer in the Department of Electronic and Electrical Engineering (EEE) before becoming a Design Engineer in industry. From 1997 to 2000, he was a Postdoctoral Researcher in the Electrical Machines and Drives Research Group, Department of EEE, University of Sheffield, where he is now a Lecturer. His research interests include electromagnetic power trains for hybrid electric vehicles, aerospace actuators, and industrial applications.

P. H. Mellor is Professor of Electrical Engineering in the Department of Electrical and Electronic Engineering, University of Bristol, Bristol, U.K. He has held academic posts at Sheffield (1990-2000) and Liverpool (1986-1990) Universities. During 1998-1999, he held a Royal Society Industrial Fellowship to research and develop electric drives for automotive and aerospace applications at the TRW-Conekt research center, Birmingham. His current research includes high-efficiency electric drives and actuation systems for application in more electric aircraft and hybrid-electric vehicles. 